\title{
Using a wavelength tunable diode laser to measure the beat length of a birefringent fiber
}

Fang-Wen Sheu, Shu-Chun Yang

Fang-Wen Sheu, Shu-Chun Yang, "Using a wavelength tunable diode laser to measure the beat length of a birefringent fiber," Proc. SPIE 9665, Tenth International Topical Meeting on Education and Training in Optics and Photonics, 96650W (3 June 2007); doi: 10.1117/12.2207339

SDIE Event: Tenth International Topical Meeting on Education and Training in Optics and Photonics, 2007, Ottawa, Ontario, Canada 


\title{
Using a wavelength tunable diode laser to measure the beat length of a birefringent fiber
}

\author{
Fang-Wen Sheu and Shu-Chun Yang \\ Department of Applied Physics, National Chiayi University, Chiayi 60004, Taiwan \\ Tel: +886-5-2717993; Fax: +886-5-2717909; E-mail: fwsheu@mail.ncyu.edu.tw
}

\begin{abstract}
In this report we demonstrated a method for measuring the beat length of a birefringent fiber. In this method the beat length is determined from the wavelength dependence of the phase difference between two orthogonally polarized modes at the output end of a sample fiber. In addition to the mode hopping of the laser diode's optical wavelength due to the temperature variation, we have also observed the phase hopping of the output light polarization at the end face of the birefringent fiber. It is a simple and precise method to determine the birefringence magnitude of anisotropic materials in an optics laboratory course.

(C) 2007 Optical Society of America

OCIS codes: (140.2020) Diode lasers; (260.1440) Birefringence; (060.2420) Fibers, polarization-maintaining
\end{abstract}

\section{Introduction}

In heterodyne-type optical communications or some kinds of fiber-optic measurement, the polarization state of the received signal must be kept constant. To meet this requirement, various birefringent fibers have been developed in which the propagation constants of two orthogonally polarized modes $\left(\mathrm{HE}_{\mathrm{x}}\right.$ and $\left.\mathrm{HE}_{\mathrm{y}}\right)$ are different. The degree of such modal birefringence is often expressed by the beat length between these two modes. There are many methods to measure the beat length. In this report, the beat length is determined from the wavelength dependence of the phase difference between the two modes at the output end of a sample fiber [1].

\section{Experimental Setup and Principle of Measurement}

Figs. 1(a) and 1(b) show the schematic diagram and photograph of the experiment setup. An AlGaInP diode laser (THORLABS DL3147-060 Sanyo) is used as the light source. As shown in Fig. 2, the optical wavelength is tunable by controlling the laser diode's temperature, and the laser spectrum is measured by an optical spectrum analyzer (ADVANTEST Q8384). The linearly polarized laser light, having a polarization angle of $45^{\circ}$ with respect to the principal axes of the birefringent sample fiber [Figs. 1(c) and 1(d), 3M FS-PM-7811, $\Delta \mathrm{n}=7.718 \times 10^{-4}$ ], is launched at the fiber input end via a microscopic objective lens [2]. The phase difference between the $\mathrm{HE}_{\mathrm{x}}$ and $\mathrm{HE}_{\mathrm{y}}$ modes at the fiber output end is given as $\phi=\ell \Delta \beta$, where $\Delta \beta=\frac{2 \pi}{\lambda} \cdot \Delta \mathrm{n}$ denotes the difference in the propagation constants of the two modes and $\ell$ is the fiber length. The phase difference $\phi$ can be determined from the maximum and minimum transmitted light intensities $\mathrm{I}_{\mathrm{a}}$ and $\mathrm{I}_{\mathrm{b}}$, respectively, of the elliptical polarization as $\sin \phi= \pm \frac{2 \sqrt{\mathrm{I}_{\mathrm{a}}}}{\mathrm{I}_{\mathrm{a}}+\mathrm{I}_{\mathrm{b}}}$, when we rotate an analyzer behind the fiber and measure the transmitted optical power. Because of the variation of the device temperature, the optical wavelength $\lambda$ of the laser light source is swept by a small amount of $\Delta \lambda$, where $|\Delta \lambda|<<\lambda$. Then the change in $\phi$ induced by $\Delta \lambda$ is given as $\Delta \phi=\ell \Delta \lambda \frac{\mathrm{d}(\Delta \beta)}{\mathrm{d} \lambda}=\ell \Delta \lambda\left(-\frac{2 \pi}{\lambda^{2}} \Delta n\right)=\ell \Delta \lambda\left(-\frac{\Delta \beta}{\lambda}\right)=-\frac{\Delta \lambda}{\lambda} \ell \Delta \beta$. Using the definition of beat length $\mathrm{L}_{\mathrm{B}}=\frac{2 \pi}{\Delta \beta}$, it can be rewritten as $\frac{\Delta \phi}{\Delta \lambda}=-\frac{1}{\lambda} \frac{2 \pi \ell}{\mathrm{L}_{\mathrm{B}}}$. Thus, by measuring the phase difference $\phi$ as a function of the wavelength $\lambda$, we can calculate the $\phi-\lambda$ relationship and hence obtain the value of beat length $\mathrm{L}_{\mathrm{B}}$.

Tenth International Topical Meeting on Education and Training in Optics and Photonics, edited by Marc Nantel, Proc. of SPIE Vol. 9665, 96650W · ( 2007 SPIE, OSA, IEEE, ICO doi: $10.1117 / 12.2207339$ 
(a)

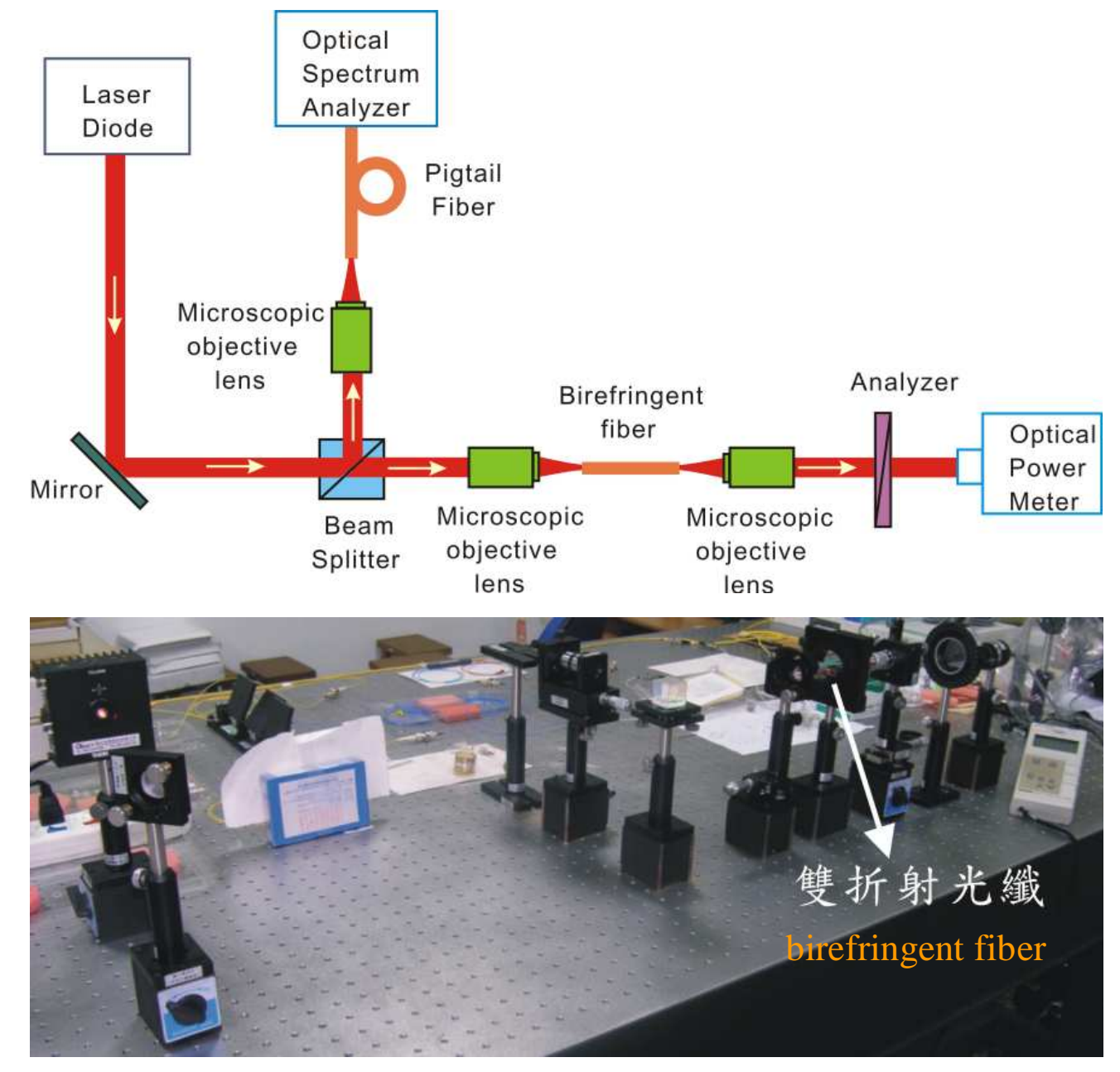

(b)

(c)

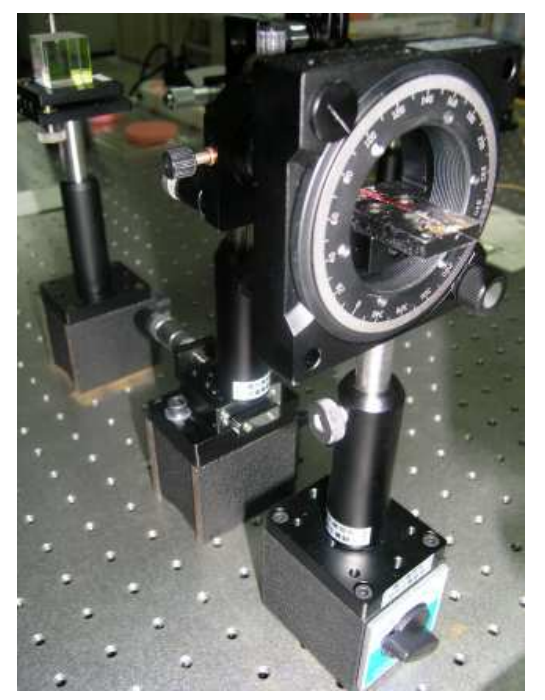

(d)



Fig. 1. The (a) schematic diagram amd (b) photograph of the experimental setup. (c) The birefringent fiber under test in a rotary mount. (d) The cross-section picture of the birefringent fiber (3M Single Mode Polarization Maintaining Fiber, FS-PM-7811, THORLABS INC. Catalog 2004). 


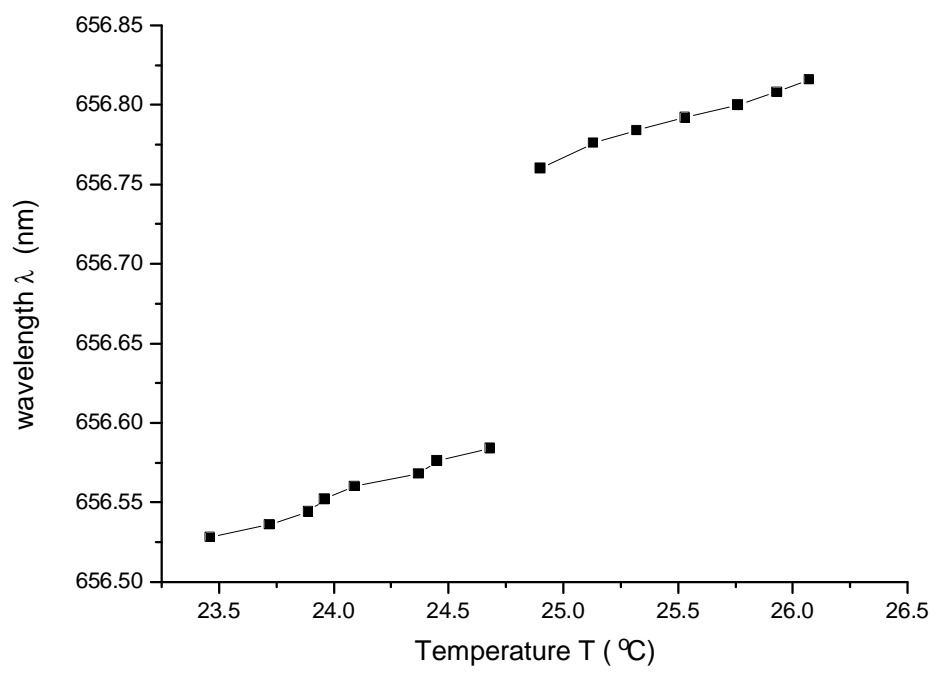

Fig. 2. The laser optical wavelength versus the laser diode's temperature.

\section{Experimental Results}

The wavelength dependence on the device temperature reveals a discontinuous change because of the mode hopping of the laser diode. As shown in Fig. 2, there are two kinds of longitudinal modes, one of which has a center wavelength $\lambda_{\mathrm{c}}=656.556 \mathrm{~nm}$ and the other has $\lambda_{\mathrm{c}}=656.79 \mathrm{~nm}$. The corresponding measured $\phi-\lambda$ diagrams also exhibit a hopping phenomenon and are shown in Figs. 3(a) and 3(b). The slopes of their linear fitting curves are $\frac{\Delta \phi}{\Delta \lambda}=-47.19 \% / \mathrm{nm}$ and $\frac{\Delta \phi}{\Delta \lambda}=-47.71 \% \mathrm{~nm}$, respectively. The predicted beat lengths $\mathrm{L}_{\mathrm{B}}=\lambda_{\mathrm{c}} /$ $\Delta \mathrm{n}$ are $0.8507 \mathrm{~mm}$ and $0.8509 \mathrm{~mm}$, respectively. The sample fiber has a length $\ell=7.2 \mathrm{~cm}$. Using the relationship $\frac{\Delta \phi}{\Delta \lambda}=-\frac{1}{\lambda_{\mathrm{c}}} \frac{2 \pi \ell}{\mathrm{L}_{\mathrm{B}}}$, we can calculate the measured beat length $\mathrm{L}_{\mathrm{B}}$ to be $0.8366 \mathrm{~mm}$ and $0.8272 \mathrm{~mm}$ for the two kinds of longitudinal modes, and the error percents are $1.657 \%$ and $2.785 \%$, respectively. The errors may be due to the device thermal fluctuation in tuning the temperature of the laser diode.

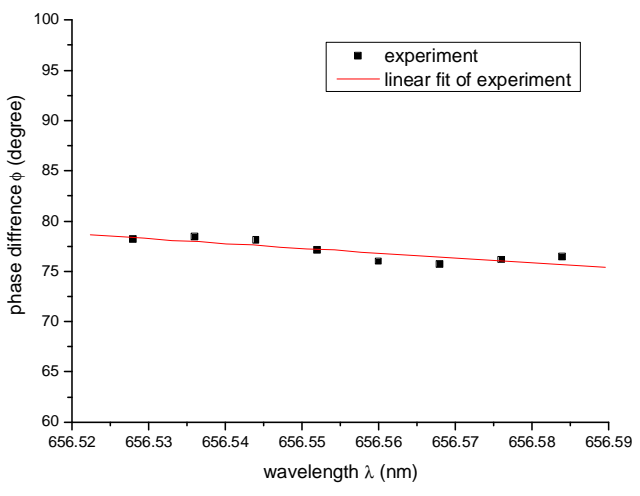

(a)

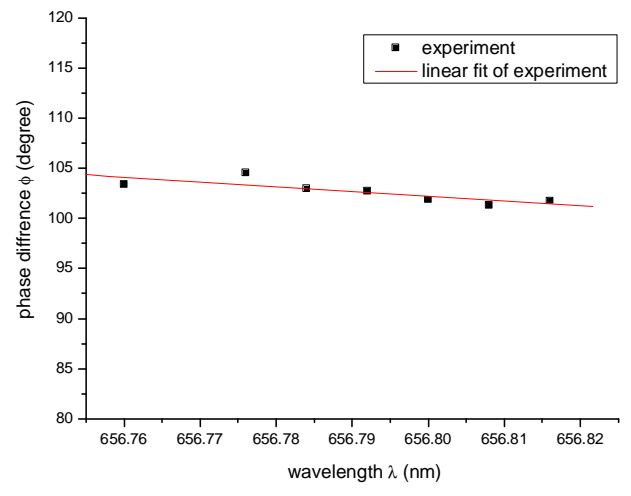

(b)

Fig. 3. The measured results and linear fits of the phase difference $\phi$ as a function of the wavelength $\lambda$ for (a) the longitudinal mode with center wavelength $\lambda_{\mathrm{c}}=656.556 \mathrm{~nm}$, and (b) another longitudinal mode with center wavelength $\lambda_{\mathrm{c}}=656.79 \mathrm{~nm}$. 


\section{Conclusion}

We have successfully achieved measuring the beat length of a birefringent fiber by a wavelength tunable diode laser. In this method the beat length is determined from the wavelength dependence of the phase difference between two orthogonally polarized modes at the output end of a sample fiber. In addition to the mode hopping of the laser diode's optical wavelength due to the temperature variation, we have also observed the phase hopping of the output light polarization at the end face of the birefringent fiber, because the fiber length is far larger than that of the birefringent retardation wave plates. In an optics laboratory course, this simple method can be used to determine the value of beat length, or the magnitude of birefringence, of any anisotropic materials precisely.

\section{Acknowledgements}

We acknowledge the financial support from the National Science Council, Taiwan, through project NSC-95-2815-C-415-003-M.

\section{References}

[1] K. Kikuchi and T. Okoshi, "Wavelength-sweeping technique for measuring the beat length of linearly birefringent optical fibers", Opt. Lett. 8, $122(1983)$

[2] Ch. Silberhorn, P.K. Lam, O. Weiß, F. König, N. Korolkova, and G. Leuchs, "Generation of Continuous Variable EinsteinPodolsky-Rosen Entanglement via the Kerr Nonlinearity in an Optical Fibre", Phys. Rev. Lett. 86, 4267 (2001). 